\title{
Non-destructive testing based on vibrations in the low to mid-frequency range
}

\author{
Philip Becht ${ }^{1,2, *}$, Elke Deckers ${ }^{1,2}$, Claus Claeys ${ }^{1,2}$, Bert Pluymers ${ }^{1,2}$, and Wim Desmet ${ }^{1,2}$ \\ ${ }^{1}$ KU Leuven, Department of Mechanical Engineering, Celestijnenlaan 300 - Box 2420, 3001 Leuven \\ (Heverlee), Belgium \\ ${ }^{2}$ DMMS lab, Flanders Make, 3001 Leuven (Heverlee), Belgium
}

\begin{abstract}
Recently, it has been shown that a Time Reversal MUltiple Signal Classification (TR-MUSIC) algorithm can be employed to detect defects in samples, which are both challenging in terms of material and in terms of geometrical complexity. This can be achieved by lowering the detection frequency as compared to most other TR and TR-MUSIC applications. In this case, the method operates in a low to mid-frequency range, where accurate models are still realizable. The method relies on the measurement of the frequency response function (FRF) between multiple excitation and sensor locations. These are gathered in a matrix, which then is decomposed in its singular vectors, serving as input for a MUSIC algorithm.

In order to improve the applicability of this method, it is shown how to adapt the algorithm, in order to reduce the number of excitation locations or the number of sensors significantly. This results in a considerable speed-up for the application of this non-destructive testing strategy. Furthermore, it is investigated numerically how the robustness of the detection result under the influence of random measurement errors behaves if the number of excitation/sensor locations is reduced.Additionally, the dependency between, the decrease of the number of excitation/sensor locations and the types of defects, which can be detected is studied.
\end{abstract}

\section{Introduction}

Vibration based non-destructive testing strategies can roughly be split into 2 groups. On the one hand side, there are those working at low frequencies, making use of the change of modal properties due to a defect. Strategies in this group are generally applicable to complex structures, but suffer from a lack of sensitivity to small damage [1]. The second group consists of high-frequency strategies, which are very powerful for the detection of small defects in test-objects with simple geometry. Unfortunately, these techniques are difficult, if not impossible to apply to complex structures, since then e.g. small variations and uncertainties in the production process can affect the vibration of the structure equally much as a potential damage. In this case, no distinction can be made between altered vibrations due to a defect or due to inaccuracies.

Recently, Becht et al.[2] presented a strategy to extend a high frequency method towards lower frequencies, allowing the detection of defects in complex systems. The idea is to

\footnotetext{
*e-mail: philip.becht@kuleuven.be
} 
work in a mid-frequency range, where the upper frequency limit is set by the accuracy of the numerical model of the test-object and the production variability. At the same time, there is a minimum frequency, which needs to be included in order to guarantee the required sensitivity to a defect. The Time Reversal MUltiple SIgnal Classification (TR-MUSIC) algorithm used is building up on the work of Fink[3] Prada et al.[4], Lehmann and Devaney[5] and Marengo et al.[6]. The basic input, is the scattered wave field measured by a finite number of sensors. This scattered wave field can be calculated by subtracting the predicted wave field in the healthy part from that measured at the potentially defected test-object. Using this strategy, Becht et al. showed numerically in [2] that internal damage in a section of an air plane wing can be detected based on only 30 excitation and 30 sensor locations, both exciting/measuring solely in the normal direction on the outer skin of the wing.

The aim of this publication is to investigate whether or not it is possible to reduce the number of excitations and/or sensors in order to lower the total testing-time. Therefore, the influence of such a reduction is first analysed theoretically in section 2 . In section 3 the derived relations are validated at a numerical example. A summary of the most important findings and concluding remarks are presented in section 4.

\section{TR-MUSIC algorithm with reduced number of excitations}

The objective in this section is to briefly present the essential steps for non-destructive testing using a TR-MUSIC algorithm and to investigate the influence of a reduced number of sensors $N$ or excitations $M$ on the quality of the detection result.

The TR-MUSIC algorithm for defect detection makes use of the wave field scattered at a $\operatorname{defect} \Psi_{s}$. Applying equation (1) (valid for one frequency), this wave field can be calculated by subtracting the wave field of the intact part $\Psi_{\text {init }}$ (a priori measured or estimated by numerical models) from the one obtained for the defected part $\Psi_{t o t}$ (measurement of the test-object) at the sensor location $x$.

$$
\Psi_{t o t}(x)=\Psi_{\text {init }}(x)+\Psi_{s}(x) .
$$

A scattering matrix $\mathbf{K}$ can be defined, where each element $(m, n)$ is filled with $\Psi_{s}$ due to excitation at position $m$ and a measurement at sensor location $n$. Marengo et al.[6] showed that $\mathbf{K}$ can be decomposed into its singular values and vectors and that the left and right hand side singular vectors associated with large singular values correspond to a propagation from the excitation array to the scatterer $\boldsymbol{\Phi}_{\mathbf{k}}$ and a propagation from the scatterer to the sensor array $\boldsymbol{\Psi}_{\mathrm{k}}$, respectively. These vectors with $1 \leq k \leq R_{0}$ are spanning the signal space, $R_{0}$ is the number of physically meaningful eigenvectors. For the case of weak point scatterers $R_{0}$ is equal to the number of scatterers, for the more complex case of finite scatterers the definition of $R_{0}$ is more challenging, since the identification of 'physically meaningful' singular vectors becomes more complicated. However, $1 \leq k \leq R_{0}$ is always selecting the singular vectors associated to the largest singular values. For the special case of a rank deficient $\mathbf{K}$, there are additional vectors $R_{0}<k \leq \min (M, N)$ having no physical meaning, but the mathematical property to be orthogonal to the signal space. These are spanning the so-called noise space.

In a TR framework, the signal space singular vectors $\boldsymbol{\Psi}_{k}$ are back-propagated from the sensor-locations, which then work as transmitters or, alternatively, $\boldsymbol{\Phi}_{k}$ is back-propagated from the excitation locations. Due to the reciprocity of $\mathbf{K}$, the wave-field peaks at the location of the scatterer. In frequency domain this is equal to finding the maximum of:

$$
I_{T R}(z)=\mathbf{g}(z)^{\mathrm{H}} \mathbf{\Phi}_{k},
$$

with a test-vector $\mathbf{g}(z)$ describing the propagation from the excitation array to a pixel location on the test-object $z$ and $I_{T R}(z)$ being the image function at $z$. 
From the orthogonality between signal and noise space, it follows that if equation (2) has a maximum, then

$$
I_{\text {noise }}(z)=\mathbf{g}(z)^{\mathrm{H}} \mathbf{\Phi}_{k} \text {, for } R_{0}<k \leq \min (M, N)
$$

is equal to zero. The MUSIC algorithm is making use of this property and calculates an image value for each $z$ based on the following equation:

$$
I(z)=\left(\sum_{k=R_{0}^{\text {meas }}+1}^{\min (M, N)}\left|\mathbf{g}(z)^{\mathrm{H}} \mathbf{\Phi}_{k}\right|\right)^{-1} .
$$

In a similar way, also an image from the sensor-side, using $\boldsymbol{\Psi}_{k}$, can be derived. Therefore, the thoughts presented in the following for an excitation-side imaging are expect to hold reversely for an imaging from sensor-side as well.

The image function used in the following is based on equation (4) but, in order to improve the image quality, an additional product over the degrees of freedom at each $z$ and over all frequency lines within the frequency range of interest is added. Furthermore, a normalisation implemented accounting for the influence of the modal behaviour, which is present in the mid-frequency range. For more detailed information on the exact implementation of the algorithm, the reader is referred to [2].

\subsection{Excitation-side TR-MUSIC with reduced number of sensors}

Under the initial assumption, that the number of sensors and excitations is at least equal, $N \leq M$, reducing the number of sensors reduces the dimension of $\mathbf{K}$ and $\min (M, N)=$ $N$. As a consequence, for a given scattered field, the noise space shrinks, which results in less redundancy in the summation of equation (4). Thus a reduced resistance to random measurement errors is expected for this scenario.

Furthermore, a condition for the singular value decomposition of $\mathbf{K}$ into the singular vectors $\boldsymbol{\Phi}_{k}$ and $\boldsymbol{\Psi}_{k}$ with the physical meaning as described previously, is that from excitation side, as well as from receiver side, the search region is fully 'illuminated'. In practice, due to the use of only a finite number of excitations/sensors this requirement is likely to be violated, but the complete 'illumination' can be achieved approximately. Naturally, reducing the number of sensors increases the inaccuracy of this approximation. In consequence, it is expected that lowering $N$ leads to errors in $\boldsymbol{\Phi}_{k}$ as well as in $\boldsymbol{\Psi}_{k}$. Therefore it is anticipated that even for excitation-side TR-MUSIC, the image quality decreases due to a reduced number of sensors.

In addition, it is possible that the number of sensors is sufficiently close to the approximation of full 'illumination' to correctly represent a simple scattering event, but the same number might be insufficient to correctly represent a complex scattered wave field. For that reason, reducing the number of sensors is expected to be most critical for the detection of complex scattering events.

\subsection{Excitation-side TR-MUSIC with reduced number of excitations}

Without a loss of generality, the thoughts presented in the previous section are applicable to the case discussed in this section, obviously, now only referring to a reduced number of excitations, while making use of the exciting array to construct the image.

Furthermore, the dimension of the vector $\boldsymbol{\Phi}_{k}$ (and hence also the dimension of $\mathbf{g}$ ) is equal to the number of excitations $M$. Consequently, a reduction of the number of excitations also reduces the dimension of $\boldsymbol{\Phi}_{k}$. Even in the case of a finite structure, where the reflections at the boundaries function as image sources and thus virtually increase the array-size of the excitation, the decrease of the dimension of $\boldsymbol{\Phi}_{k}$ still reduces the image quality. This is because the reflections will arrive at the scatterer with a lower amplitude than the incident signal [3]. 


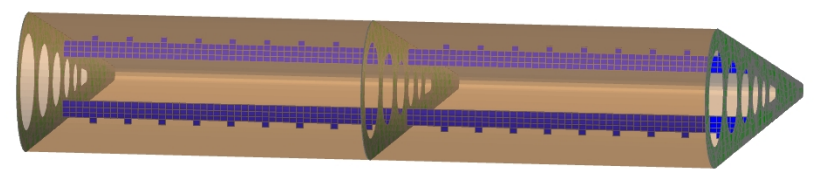

Figure 1: Model of a section of an air plane wing consisting of stiffeners (green), stingers (blue) and skin (translucent brown)

\section{Parameter study}

In this section, the expectations, which are discussed in section 2 are verified based on the numerical example of a section of an air plane wing presented in figure 1. It consists of aluminium stiffeners (green) and stingers (blue) surrounded by a composite skin (translucent brown). The 2 defect scenarios that are considered here are (1) a single defect represented by disconnecting the top right stinger from the right stiffener and (2) a double defect consisting of one defect modelled as disconnected stinger bottom left from the left stiffener and a removed connection between the top left stinger and the central stiffener. For all images, $R_{o}=4$ is applied.

As the defects are covered by the skin and as such neither visible, nor accessible, randomly selected excitation and sensor locations normal to the skin are used as inputs for the TR-MUSIC algorithm.

All images shown in figures 2 and 3 are generated based on a excitation-side imaging procedure. Hence, reducing the number of sensors reduces the number of singular values, but does not affect the number of noise-space singular vectors, which are used by the algorithm. Following the same logic, reducing the number of excitation locations does reduce both, the number of singular values, as well as the number of noise-space singular vectors.

As reference, an image for both defect scenarios is generated using 30 excitations and 30 sensors, once without errors (figure $2 \mathrm{a}$ and figure $3 \mathrm{a}$ ) and once with an artificially added random measurement error of $\mathrm{SNR}=5$ (figure $2 \mathrm{~b}$ and figure $3 \mathrm{~b}$ ). In all these scenarios, the correct zone is highlighted as defected.

In order to verify the expected deterioration of the image due to the reduction of singular values, the number of sensors is gradually reduced from 30 to 6 . However, due to the limited space, only the images corresponding to the extreme values of 30 and 6 are presented, although more in-between steps were calculated and analysed. As can be seen from the comparison of figure $2 \mathrm{a}$ and $\mathrm{c}$ or figure $3 \mathrm{a}$ and $\mathrm{c}$, there is no significant influence of this change on the image in the scenario without noise. If random noise with a level of SNR $=5$ is considered, the image quality degrades slightly - as predicted - due to the reduced redundancy in the model. This can be observed by comparing figure $2 \mathrm{~b}$ and $\mathrm{d}$ or figure $3 \mathrm{~b}$ and $\mathrm{d}$, respectively. In consequence, it can be confirmed that the reduction of an element (excitation or sensor), of which the singular vectors are not used for the TR-MUSIC algorithm, has almost no influence in the absence of errors. In contrast at the presence of random errors, a higher number of sensors is beneficial, since the errors can be partially 'filtered' out due to the redundancy added by the additional elements.

Comparing figure $2 \mathrm{e}$ to a and $\mathrm{c}$ or figure $3 \mathrm{e}$ and $\mathrm{a}$ and $\mathrm{c}$ for the noiseless scenario or figure $2 \mathrm{f}$ to $\mathrm{b}$ and $\mathrm{d}$ or figure $3 \mathrm{f}$ to $\mathrm{b}$ and $\mathrm{d}$ for the scenario with random errors, shows that the decrease of image quality is worse if the number of excitation locations is decreased, then if the number of sensors is decreased. Furthermore, it can be concluded that in case of complex scattering the decrease of the image quality due to a reduction of the excitation locations is more severe (Comparison of figure 3, 2 defects and figure 2, 1 defect). 


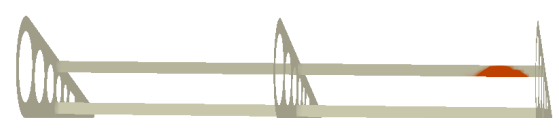

(a) no noise - 30 excitations - 30 sensors

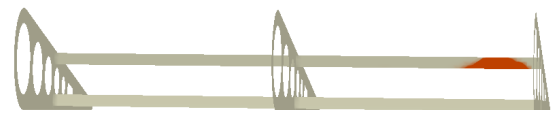

(c) no noise - 30 excitations - 6 sensors

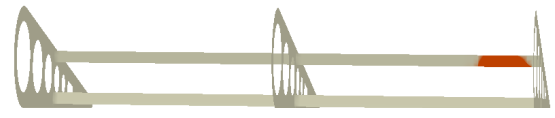

(e) no noise - 6 excitations - 30 sensors

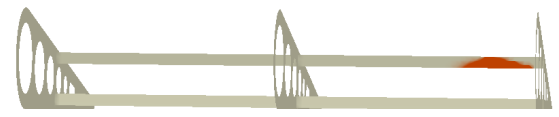

(g) no noise - 6 excitations - 6 sensors

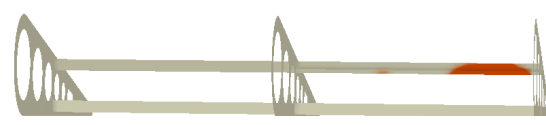

(b) $\mathrm{SNR}=5-30$ excitations -30 sensors

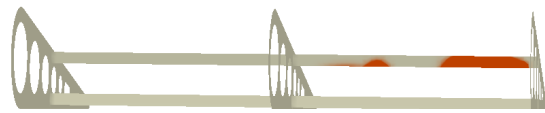

(d) $\mathrm{SNR}=5-30$ excitations -6 sensors

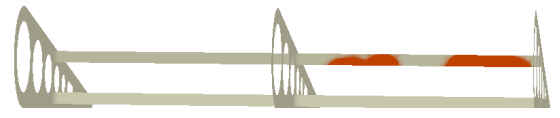

(f) $\mathrm{SNR}=5-6$ excitations -30 sensors

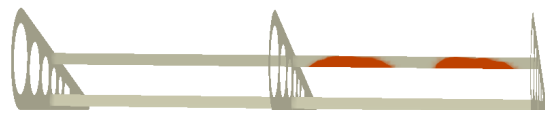

(h) $\mathrm{SNR}=5-6$ excitations -6 sensors

Figure 2: Influence of the number of sensors/excitations on the detection of 1 defect (top right stinger - right)

Figure $2 \mathrm{~g}$ shows the imaging result based on 6 sensors and 6 excitation locations, without random errors. In this case the image is comparable to those obtained in figure $2 \mathrm{c}$ and $\mathrm{e}$, where the number of sensors and excitation locations is reduced separately. The image generated based on 6 sensors and 6 excitation locations at the presence of random noise is presented in figure $2 \mathrm{~h}$. This imaging result is worse than the one obtained with only a reduced number of sensors (figure 2d), but comparable to the one obtained with only a reduced number of excitations (figure 2f). This similarity, once more supports the expectation that the imaging result is mainly driven by the number of excitations and is less influenced by the number of sensors. The same observation can be made for the case of 2 defects and 6 sensors and 6 excitations, of which the results are not presented in this paper due to the page limit.

The final prospect mentioned in section 2, a growing number of necessary elements with increasing complexity of the scattering event becomes particularly obvious when comparing the noiseless imaging results with reduced number of excitations for 1 and 2 defects. While the zone of defect in the simpler scattering event ( 1 defect in figure $2 \mathrm{e}$ ) is still predicted correctly, the imaging fails for the more complex case of 2 defects (figure $3 \mathrm{e}$ ). This confirms the expected behaviour.

\section{Conclusion}

In this article a TR-MUSIC algorithm is used to generate an image indicating defects in a complex structure. This can be done using the singular vectors of a measured scattering matrix corresponding to the excitations or to the sensors, respectively. The focus in this publication is to investigate how the quality of the image changes depending on the number of excitations and sensors. Therefore, firstly, the influence of reducing the number of excitations/sensors is deduced theoretically, with the following result:

- If the image is generated based on the excitation-side singular vectors, a reduction of the number of sensors has only a very limited effect on the image. The influence increases with increasing measurement noise due to a loss in redundant information. 


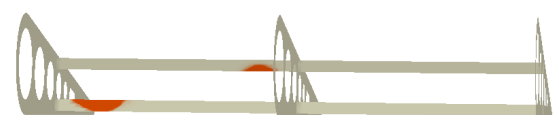

(a) no noise - 30 excitations - 30 sensors

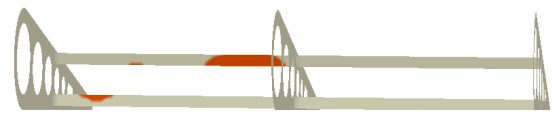

(c) no noise - 30 excitations - 6 sensors

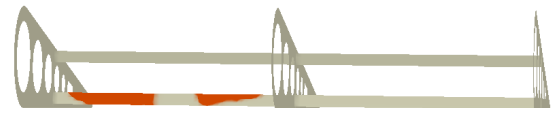

(e) no noise - 6 excitations - 30 sensors

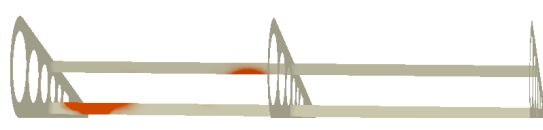

(b) $\mathrm{SNR}=5-30$ excitations -30 sensors

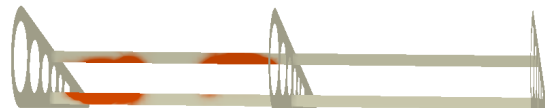

(d) SNR $=5-30$ excitations -6 sensors

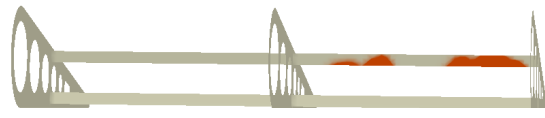

(f) $\mathrm{SNR}=5-6$ excitations -30 sensors

Figure 3: Influence of the number of sensors/excitations on the detection of 2 defects (bottom left stinger - left and top left stinger - right)

- If the image is generated based on the excitation-side singular vectors, a reduction of the number of excitations leads to a much more severe deterioration of the image. This dependency becomes even stronger with increasing complexity of the scattered field.

The same observations are expected to hold reversely for a sensor-side image with reduced number of excitations/sensors.

In a second step, the predicted behaviour for excitation-side imaging is successfully verified at the numerical example of a section of an air plane wing with 1 or 2 defects, respectively.

By applying the conclusions drawn from this publication, e.g. the physical testing time for non-destructive evaluation using a TR-MUSIC algorithm can be reduced. To achieve this, for sensor-side imaging, the number of sensors should be kept high, while the number of excitation locations should (depending on the concrete problem at hand) be reduced to a minimum.

The European Commission is gratefully acknowledged for their support of the ANTARES project (GA606817). Elke Deckers is a postdoctoral fellow of the Research Foundation - Flanders (FWO). Also the Research Fund KU Leuven is gratefully acknowledged for its support.

\section{References}

[1] Y. Zou, L. Tong, G.P. Steven, Journal of Sound and vibration, 230, 357-378 (2000).

[2] P. Becht, E. Deckers, C. Claeys, B. Pluymers, W. Desmet, Journal of Nondestructive Evaluation, under review (2018).

[3] M. Fink, Journal of Physics: Conference Series, 124(1) (2008).

[4] C. Prada, S. Manneville, D. Spoliansky, M. Fink, The Journal of the Acoustical Society of America, 99(4), 2067-2076 (1996).

[5] S.K. Lehman, A.J. Devaney, The Journal of the Acoustical Society of America 113(5), 2742-2753 (2003).

[6] E.A. Marengo, F.K. Gruber, F. Simonetti, IEEE Transactions on image processing, 16(8), 1967-1984 (2007). 